\title{
AVANT-PROPOS DU TOME III
}

Le tome III se divise en trois parties: paroi thoracique, cavités pleurales et médiastin. Lorsque les rapports topographiques et fonctionnels le nécessitent, la description déborde les limites anatomiques et s'étend aux zones de passage et aux territoires de transition. Ainsi, par exemple, à propos de la glande mammaire, l'accent est mis non seulement sur le groupe des ganglions lymphatiques axillaires mais sur la topographie du creux axillaire. Le système lymphatique et le système nerveux autonome sont traités plus en détail. Le premier, par suite de son importance clinique croissante, le second, parce qu'il a son origine dans la région thoracique. Dans le choix des illustrations, je me suis efforcé de montrer autant que possible toutes les régions mentionnées dans le texte. Les radiographies m'ont été aimablement prêtées par le Prof. K. Mülly, le Dr A. Rüttimann, P.D., le Dr T. Kiss, P.D., les Drs J. Balint, K. Kett et W. Wirth, les clichés de bronchoscopie, par le Dr R. Hug. Qu'ils trouvent ici l'expression de ma reconnaissance. Je suis redevable des dissections illustrées par la figure 9 au

Zurich, février 1968.
Dr M. Fueter, par les figures 23 et 24 , à H. Füllemann, étudiant en médecine, par la figure 8 , à $\mathrm{M}$. R. Gangel. C'est aux procédés de conservation et aux larges connaissances techniques de ce dernier que je dois d'avoir travaillé sur des pièces anatomiques demeurées dans un état remarquable.

Mes remerciements vont à Mme Hildegard Resch, ma laborantine, pour son intérêt à la tâche, le soin apporté à la transcription du manuscrit et à l'établissement de l'index alphabétique.

M. et Mme Drews-Horn ont réalisé avec une grande fidélité les dessins d'après mes esquisses. Je remercie chaleureusement $M$. W. Brandenberger, chef du Laboratoire AgfaGevaert, S.A., pour son aide et ses conseils techniques.

Le Dr H. Müntener m'a été d'un grand secours dans la rédaction de certaines parties du texte et dans la correction du manuscrit. Mes remerciements s'adressent enfin au Dr Ch. Syburra et à Mlle M. Ernst pour leur collaboration dans la correction de l'ouvrage et l'établissement de l'index alphabétique.

\section{Dr Stefan KUBIK}

Professeur d'Anatomie à 1'Université de Zurich 
